\title{
Laser Melting in Liquids Using Gold Nanoparticles Stabilized by $\mathrm{Na}_{2} \mathrm{CO}_{3}$
}

\author{
Takeshi Tsuji $^{* 1}$, Isami Takade ${ }^{* 1}$, Masaharu Tsuji $^{* 2}$, Yoshie Ishimawa ${ }^{* 3}$, Naoto Koshizaki ${ }^{* 4}$ \\ ${ }^{*}$ Interdisciplinary Graduate School of Science and Engineering, Shimane University, 1060 Nishikawatsu-Cho, \\ Matsue 690-8504 Japan \\ E-mail:tkstsuji@riko.shimane-u.ac.jp \\ ${ }^{* 2}$ Institute of Materials Chemistry and Engineering, Kyushu University, Kasuga, Fukuoka 816-8580, Japan \\ ${ }^{* 3}$ Nanosystem Research Institute, National Institute of Advanced Industrial Science and Technology, \\ Tsukuba, Ibaraki 305-8565, Japan \\ ${ }^{* 4}$ Factory of Engineering, Hokkaido University, Sapporo, Hokkaido 001-0020, Japan
}

\begin{abstract}
We have been showing that laser melting in liquids (LML) using gold nanoparticles (AuNPs) stabilized by reagents is a promising technique to fabricate submicron-sized spherical particles of gold (AuSMPs) efficiently. In the present study, we prepared AuSMPs using $\mathrm{Na}_{2} \mathrm{CO}_{3}$, from which no toxic side product will be produced by laser irradiation, as a stabilizing reagent. The result showed that AuSMPs were successfully obtained by adjusting the concentration of $\mathrm{Na}_{2} \mathrm{CO}_{3}$. By comparing the size and the formation process of AuSMPs with those observed for LML using AuNPs stabilized by citrate and $\mathrm{NaCl}$, it was found that the size of AuSMPs and the agglomeration and fusion process of AuNPs depends on the reagent species markedly.
\end{abstract}

DOI: $10.2961 / \mathrm{jlmn} .2015 .03 .0017$

Keywords: submicron-sized particles, ligands, colloids, agglomeration, laser ablation

\section{Introduction}

The size control of metal particles is an essential subject in colloid science, because various properties of particles depends on their size markedly. Laser irradiation for colloidal particles has been a promising size control technique, because of the simplicity of the procedure.

Recently, laser melting in liquids (LML), in which nonfocused laser irradiation at moderate fluence is conducted to induce the colloidal particle fusion, attracts much attention as a novel size control technique [1-9]. It is remarkable that submicron-sized spherical particles are prepared as a result of the colloidal particle fusion. The submicronsized spherical particles (SMPs) produced by LML must be good candidates of cell markers and light scattering medium due to their smooth surface and narrow size distribution. For example, we have demonstrated that a random laser system can be constructed using $\mathrm{ZnO}$ SMPs prepared by LML [10].

In our previous study, gold SMPs (AuSMPs) were prepared by LML using source colloidal gold nanoparticles (AuNPs) stabilized by citrate [8] and $\mathrm{NaCl}$ [11]. These stabilizing reagents have been employed to prevent the significant sedimentation of the AuNPs prior to and during LML which is observed when AuNPs dispersed in pure water are used as the source AuNPs. The results showed that 1) although the AuNPs sedimentation should be reduced, the AuNP agglomeration is necessary to generate AuSMPs, because AuSMPs are formed via the fusion of the agglomerating AuNPs. 2) When AuNPs stabilized by these reagents are irradiated by laser, the stabilizing reagents on the AuNPs are removed from the surface due to the laser heating of the AuNPs, leading to the AuNP agglomeration. This laser-induced agglomeration of AuNPs stabilized by reagents is significantly usable: first, the stable source AuNPs can be prepared with suppressing the sedimentation prior to LML, then the AuNPs agglomeration and fusion can be induced by laser irradiation simultaneously during LML. Another important result obtained by the previous studies was that 3 ) the size and the formation process of AuSMPs were different between LML using citrate and that using $\mathrm{NaCl}$ as the stabilizing reagents.

These findings indicate that stabilizing reagent is an important factor which influence on the formation efficiency and the size of AuSMPs in LML. Therefore, further clarification of the roles of stabilizing reagent in LML is necessary. In the present study, we have prepared AuSMPs by LML using $\mathrm{Na}_{2} \mathrm{CO}_{3}$ as a stabilizing reagents. Our preliminary experiments showed that the stability of AuNPs prepared by laser ablation in aqueous solutions of $\mathrm{Na}_{2} \mathrm{CO}_{3}$ is higher than that of AuNPs prepared in pure water, and is increased with the increasing $\mathrm{Na}_{2} \mathrm{CO}_{3}$ concentration, indi-

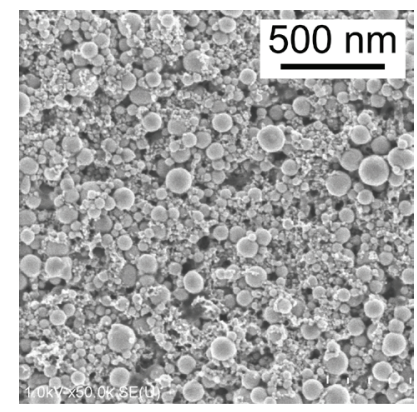

Fig. 1 SEM image of AuNPs prepared by laser ablation in a 0.05 $\mathrm{mM} \mathrm{Na} \mathrm{CO}_{3}$ aqueous solution. 


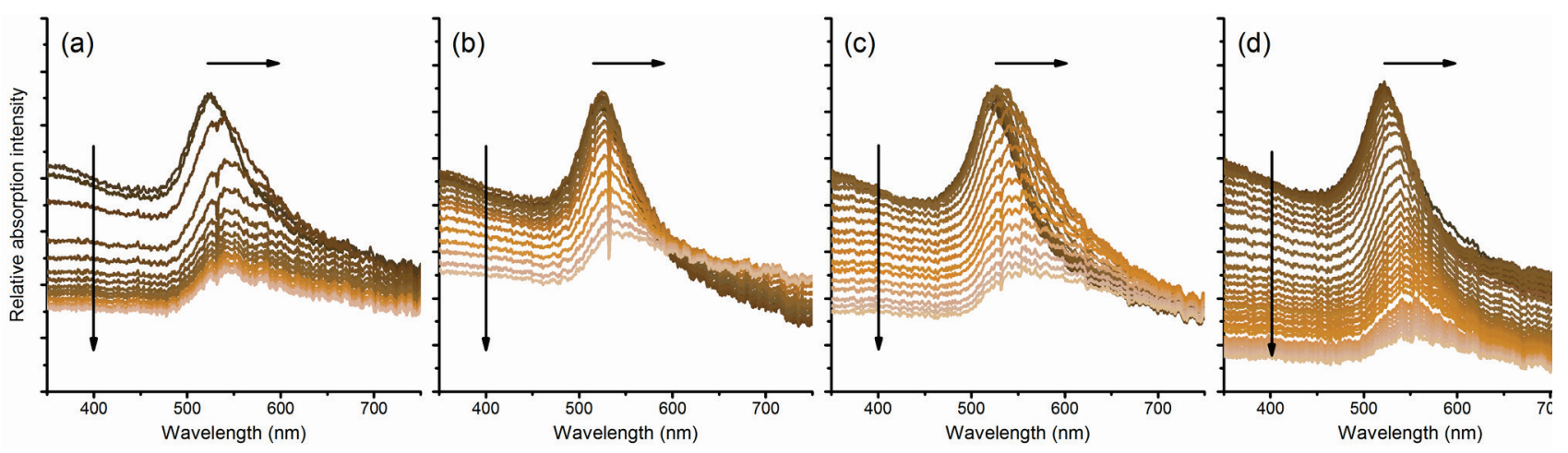

Fig. 2 Temporal changes in absorption spectra during LML of AuNPs stabilized by (a) $0.05 \mathrm{mM}$, (b) $0.1 \mathrm{mM}$, (c) $0.3 \mathrm{mM}$, (d) 0.5 $\mathrm{mM} \mathrm{Na} \mathrm{CO}_{3}$. The laser fluence used in LML was $60 \mathrm{~mJ} / \mathrm{cm}^{2}$. The interval of each spectrum is $1 \mathrm{~min}$.

cating that $\mathrm{Na}_{2} \mathrm{CO}_{3}$ can act as a stabilizing reagent of AuNPs prepared by LAL. It is also important that, similarly to $\mathrm{NaCl}$, no toxic side product, which can be generated by laser irradiation for organic reagents such as citrate, will be generated as a result of laser irradiation for $\mathrm{Na}_{2} \mathrm{CO}_{3}$.

\section{Experimental}

As shown by the previous works [11-14], salts can act as a stabilizing reagent when they are present in the solution during LAL, because they can be adsorbed on "clean" surface of AuNPs just after the generation, although when salts are added to AuNPs colloids prepared in pure water, they induce the sedimentation (salting out).

The AuNPs stabilized by $\mathrm{Na}_{2} \mathrm{CO}_{3}$ were prepared by the laser ablation in liquids (LAL) technique. LAL was conducted for a piece of gold plate immersed in $5 \mathrm{~mL}$ of aqueous solution of $\mathrm{Na}_{2} \mathrm{CO}_{3}$ in a glass cell using the focused laser beam of a nanosecond-pulse Nd:YAG laser (GCR200; Spectra-Physics KK). The wavelength, pulse duration, and repetition rate were, respectively $1064 \mathrm{~nm}, 10 \mathrm{~ns}$, and $10 \mathrm{~Hz}$. The typical laser fluence at the focal point was 36 $\mathrm{J} / \mathrm{cm}^{2}$. LML was conducted for $3 \mathrm{~mL}$ of the colloidal AuNPs solution in a glass cell using a non-focused laser beam of another nanosecond-pulse Nd:YAG laser (GCR100; Spectra-Physics KK). The wavelength, pulse duration, repetition rate were, respectively $532 \mathrm{~nm}, 10 \mathrm{~ns}$, and $10 \mathrm{~Hz}$. The non-focused laser beam diameter was $5 \mathrm{~mm}$. The colloidal solutions were stirred during LML using a magnetic stirrer. The products were analyzed using a UV-Vis spectrometer (UV-2450; Shimadzu Corp.) and a scanning electron microscope (SEM, SU8000; Hitachi Ltd.). To investigate the agglomeration-fusion dynamics of AuNPs, we carried out in situ observation of the absorption spectra of $\mathrm{Au}$ colloids during LML. To do this, the probe light of a Xenon lamp, which was transmitted through the cell containing AuNP colloidal solution, was detected using a multi-channel spectrometer (PMA-10; Hamamatsu Photonics K.K.).

\section{Results and discussion}

\subsection{Preparation of AuSMPs using $\mathrm{Na}_{2} \mathrm{CO}_{3}$}

In the present experiments, the concentration of $\mathrm{Na}_{2} \mathrm{CO}_{3}$ in AuNPs was adjusted at $0.05 \mathrm{mM}$ and higher. Below 0.05 $\mathrm{mM}$, the AuNP sedimentation during LAL and LML became significant. The stabilization mechanism of $\mathrm{Na}_{2} \mathrm{CO}_{3}$ has not been clarified in the present study. However, considering the information provided previous reports that negative ions are usually adsorbed on AuNPs prepared by laser ablation in aqueous solutions of salts [13,14], it is suggested that $\mathrm{CO}_{3}{ }^{2-}$ ions will be adsorbed on the surface of AuNPs during the formation process (laser ablation process), and provide supplemental negative charge to colloidal AuNPs. A SEM image of AuNPs prepared in $0.05 \mathrm{mM}$ $\mathrm{Na}_{2} \mathrm{CO}_{3}$ is shown in Fig. 1. AuNPs of ca. $5 \sim 150 \mathrm{~nm}$ diameter are observed. Fig. 2(a) (d) show temporal changes of absorption spectra of $\mathrm{Au}$ colloids in $\mathrm{Na}_{2} \mathrm{CO}_{3}$ aqueous solutions at the concentrations of $0.05 \sim 0.5 \mathrm{mM}$ during LML. As a result of laser irradiation, the plasmon bands of Au colloids were red-shifted and broadened. Such spectral changes were similar to those observed in LML using citrate [8] and those observed in $\mathrm{LML}$ using $\mathrm{NaCl}$ [11], indicating that the increase in the $\mathrm{Au}$ particle size due to the agglomeration and fusion of colloidal particles, i.e., the formation of AuSMPs, were induced by laser irradiation.

In Fig. 3, the peak position of the plasmon bands in the absorption spectra of Au colloids during LML (Fig. 2) is plotted as a function of laser irradiation time. As mentioned in the former part, the red-shift of the plasmon bands indicates the size increase of $\mathrm{Au}$ colloids due to the laserinduced agglomeration and fusion. Two remarkable futures were observed in these plots. First, the plot (b), (c), and (d) have an "induction period" in which no red-shift occurs at the beginning of laser irradiation. After the induction period, the red-shift occurs steeply. Second, the duration of the induction period increases with the increasing $\mathrm{Na}_{2} \mathrm{CO}_{3}$ concentration. It is reasonable to consider that the plot (a) contains an induction period shorter than $1 \mathrm{~min}$. Such behaviors of the agglomeration and fusion of $\mathrm{Au}$ colloids are similar to those observed in LML using citrate [8] and are different from those observed in $\mathrm{LML}$ using $\mathrm{NaCl}$ [11] as

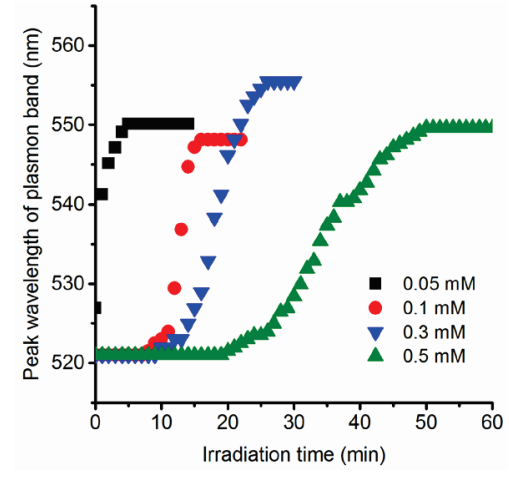

Fig. 3 The peak positions of the plasmon bands of Au colloids shown in Figs. 2(a) (d) plotted as a function of irradiation time. 
(a)

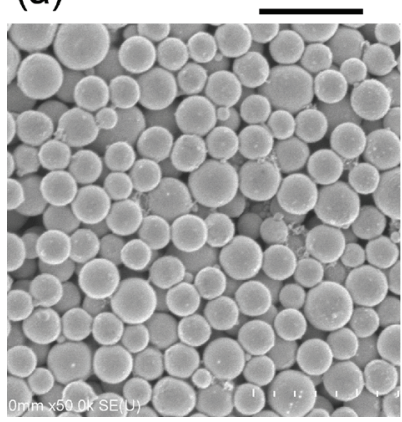

(b)

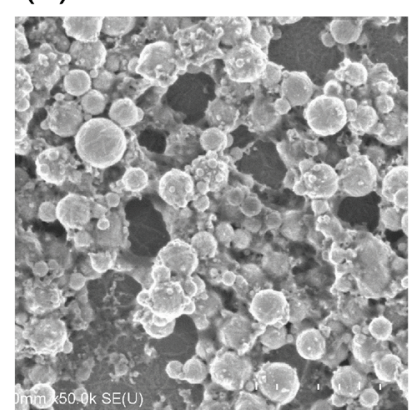

(c)

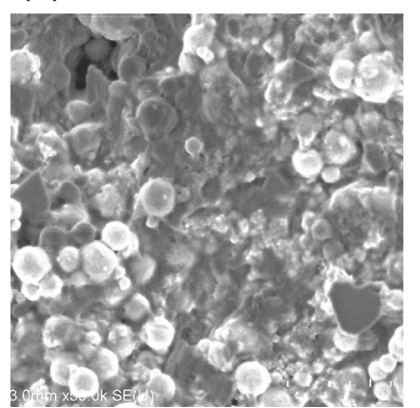

(d)

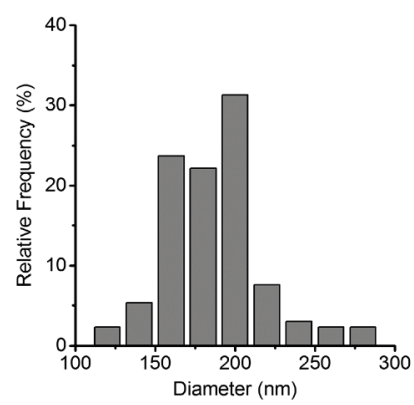

Fig. 4 SEM images of AuSMPs prepared by LML of AuNPs stabilized by (a) $0.05 \mathrm{mM}$, (b) $0.1 \mathrm{mM}$, (c) $0.3 \mathrm{mM} \mathrm{Na}_{2} \mathrm{CO}_{3}$. The laser fluence used in LML was $60 \mathrm{~mJ} / \mathrm{cm}^{2}$. The durations of LML were adjusted for (a) $15 \mathrm{~min}$, (b) $25 \mathrm{~min}$, (c) $30 \mathrm{~min}$. (d) The size distribution of AuSMPs obtained from (a). The average diameter of AuSMPs in (a) is $185 \mathrm{~nm}$.

will be discussed later (Fig. 6).

Fig. 4(a), 4(b), and 4(c) respectively shows SEM images of AuSMPs produced by LML in $\mathrm{Na}_{2} \mathrm{CO}_{3}$ solutions at the concentrations of $0.05,0.1$, and $0.3 \mathrm{mM}$. Above $0.3 \mathrm{mM}$ $\mathrm{Na}_{2} \mathrm{CO}_{3}$, SEM observation was difficult because of remaining of significant amount of $\mathrm{Na}_{2} \mathrm{CO}_{3}$ covering $\mathrm{Au}$ particles. The AuSMPs with diameter of $\sim 180 \mathrm{~nm}$ were observed in all the samples as a result of laser irradiation, consistent with the spectral changes. The results shown by Figs. 2 4 indicate that AuSMPs can be prepared by LML using AuNPs stabilized by $\mathrm{Na}_{2} \mathrm{CO}_{3}$.

A remarkable future found by comparing Fig. 3 with Fig. 4 is that the amount of AuNPs remained after LML is increased with the increasing induction period. In addition, the same relation between the amount of remaining AuNPs and the duration of the induction period was observed when LML was carried out using the source colloids containing higher relative concentration of AuNPs. Fig. 5(a) shows the temporal change of the peak position during laser irradiation for AuNPs in a $0.1 \mathrm{mM} \mathrm{Na} \mathrm{CO}_{3}$ solution that were prepared by LAL for $20 \mathrm{~min}$ (the AuNPs used to prepare AuSMPs in Fig. 4(b) were prepared by LAL for 10 min in a $0.1 \mathrm{mM} \mathrm{Na} \mathrm{CO}_{3}$ solution). The concentration of AuNPs in this solution was higher by ca. 1.8 than that of AuNPs used to prepare AuSMPs shown in Fig. 4(b), from the comparison of absorption intensity. The duration of the induction period in Fig. 5(a) was reduced to less than $1 \mathrm{~min}$. In a SEM image of AuSMPs obtained by this experiment (Fig. 5(b)), the amount of remaining AuNPs after LML is significantly reduced, compared with Fig. 4(b). Therefore, these results indicate that fast agglomeration of the source AuNPs reduces the remaining of the AuNPs after LML. This finding is significantly important to increase the formation efficiency of AuSMPs and to control the particles size precisely, while further study is necessary to explain this phenomenon.

\subsection{Comparison with preparations of AuSMPs using citrate and $\mathrm{NaCl}$}

In Fig. 6, the temporal changes in the peek position of the plasmon band of Au colloids stabilized by citrate, $\mathrm{NaCl}$, and $\mathrm{Na}_{2} \mathrm{CO}_{3}$ during laser irradiation are compared. It is notable that behaviors of the agglomeration and fusion of AuNPs stabilized by $\mathrm{Na}_{2} \mathrm{CO}_{3}$ are similar to those observed in LML using AuNPs stabilized by citrate and are different from those observed in LML using AuNPs stabilized by $\mathrm{NaCl}$.

As reported previously [8], the formation process of AuSMPs in LML using citrate can be proposed as shown in Fig. 7(a). Before laser irradiation, AuNPs prepared by LAL in citrate solutions adsorb citrate ions on the surface and are stabilized. These citrate ions are decomposed by laser irradiation because of laser heating of AuNPs. The number (a)

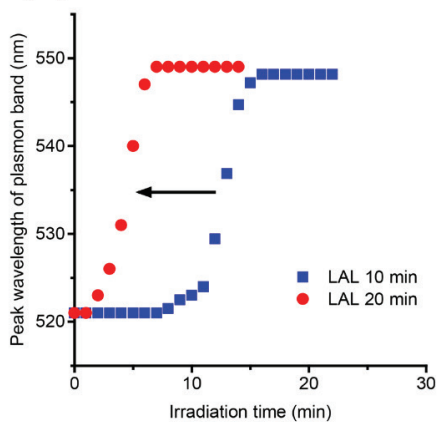

(b)

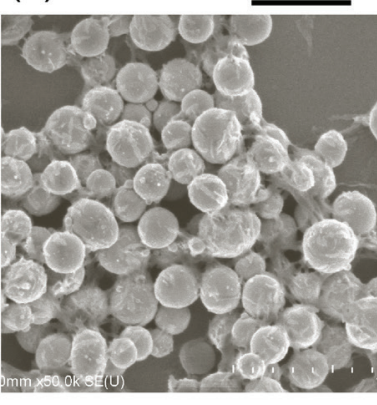

Fig. 5 (a) Temporal changes in the peak position of the plasmon band during LML of AuNPs prepared in $0.1 \mathrm{mM}$ $\mathrm{Na}_{2} \mathrm{CO}_{3}$ for $10 \mathrm{~min}$ and 20 min. (b) SEM image of AuSMPs obtained from AuNPs prepared in $0.1 \mathrm{mM} \mathrm{Na} \mathrm{CO}_{3}$ for 20 min. The laser fluence used in LML was $60 \mathrm{~mJ} / \mathrm{cm}^{2}$. The average diameter of the AuSMPs are $197 \mathrm{~nm}$.

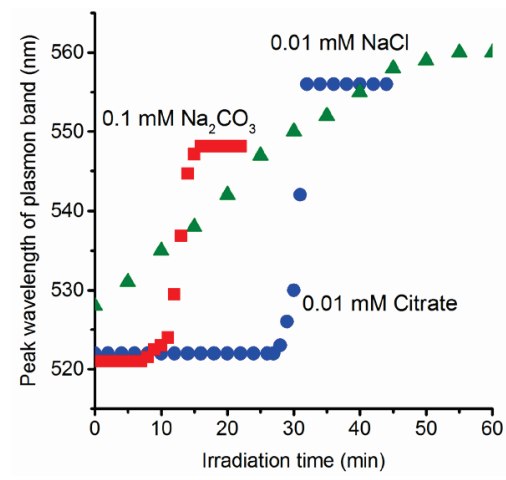

Fig. 6 Comparison of temporal changes in the peak position of the plasmon bands during LML using AuNPs in $0.1 \mathrm{mM}$ $\mathrm{Na}_{2} \mathrm{CO}_{3}, 0.01 \mathrm{mM}$ sodium citrate, $0.05 \mathrm{mM} \mathrm{NaCl}$. The laser fluence used in LML was $60 \mathrm{~mJ} / \mathrm{cm}^{2}$. These curves are plotted to show typical behavors of the agglomeration-fusion process of AuNPs stabilized by these reagents. Duration of the induction period depends on the reagents concentration. 

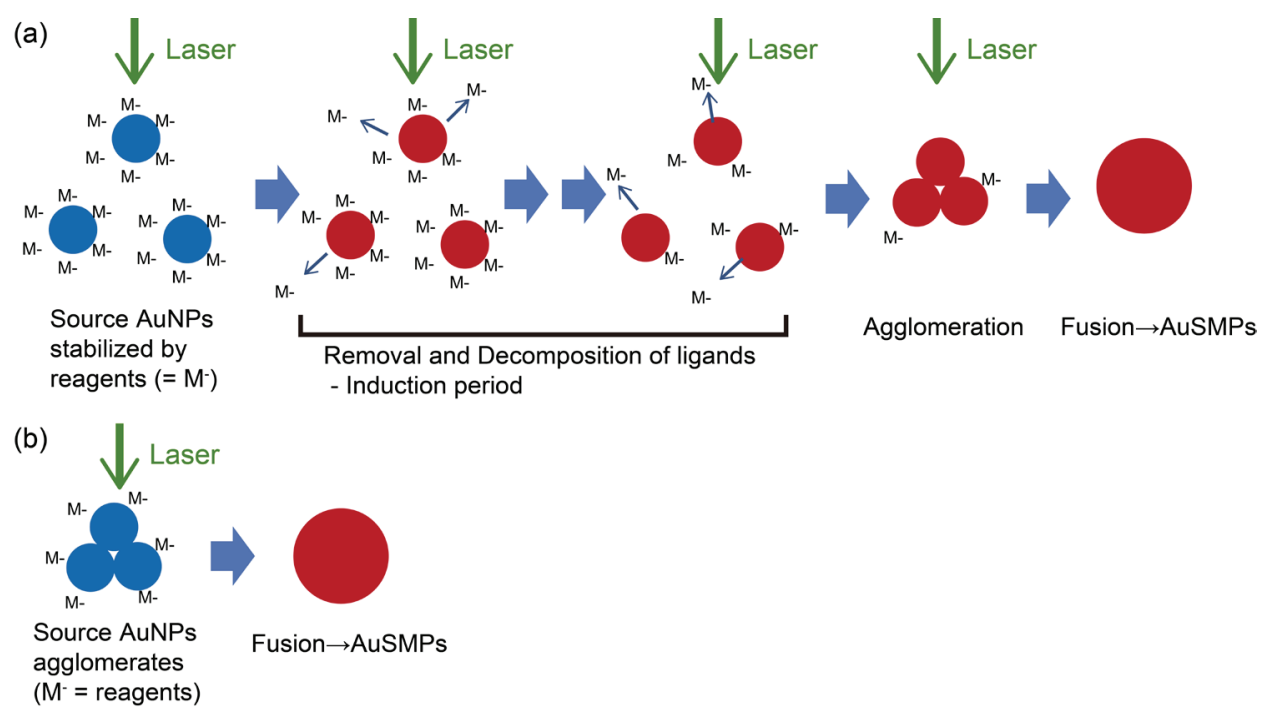

Fig. 7 Possible formation processes of AuSMPs in LML using AuNPs stabilized by (a) citrate and $\mathrm{Na}_{2} \mathrm{CO}_{3}$ and (b) $\mathrm{NaCl}$. Reagent molecules are indicated as $\mathrm{M}^{-}$.

of citrate ions on AuNPs is decreased with the increasing laser irradiation time, and finally the number of citrate ions on AuNPs becomes insufficient to stabilize the AuNPs. The induction period observed in the red-shift of plasmon bands can be interpreted as the period in which the number of citrate molecules are decreasing before the AuNP agglomeration begins. After the induction period, the AuNPs agglomeration and fusion occur, leading to the AuSMP formation.

On the other hand, no induction period was observed in LML using AuNPs stabilized by $\mathrm{NaCl}$. In $\mathrm{NaCl}$ solutions, it is suggested that AuNPs agglomerates are formed before laser irradiation, and that AuSMPs are formed directly from the AuNP agglomerates (Fig. 7(b)) [11]. Actually, in the absorption spectra of $\mathrm{Au}$ colloids before laser irradiation (Fig. 8), the relative absorption intensity in in the 550--800 $\mathrm{nm}$ region of the plasmon band, where light scattering component by AuNPs larger than $100 \mathrm{~nm}$ are mainly contained [15], is higher in a $\mathrm{NaCl}$ solution than in a citrate solution. Another difference between the agglomerationfusion behaviors between LML using citrate and LML using $\mathrm{NaCl}$ is that the AuNPs agglomeration and fusion in $\mathrm{NaCl}$ solutions occur more slowly than those occur in citrate solutions. The slower agglomeration and fusion will occur because $\mathrm{NaCl}\left(\mathrm{Cl}^{-}\right)$ligands are not decomposed by laser irradiation. After the mechanical removal from AuNPs by laser irradiation ( $\mathrm{NaCl}$ will be removed from AuNPs due to the generation of shockwaves by laser heating of AuNPs.), $\mathrm{NaCl}\left(\mathrm{Cl}^{-}\right)$can be re-adsorbed onto AuNPs and can disturb the AuNPs agglomeration, resulting in the lowering in the agglomeration rate.

Taking these interpretation of the results of the previous studies into account, the fact that behaviors of the agglomeration-fusion process observed in LML using $\mathrm{Na}_{2} \mathrm{CO}_{3}$ are similar to those observed in LML using citrate, while they are different from those observed in LML using $\mathrm{NaCl}$ suggests that 1) the formation of AuSMPs in $\mathrm{Na}_{2} \mathrm{CO}_{3}$ solutions begins from dispersed AuNPs and laser-induced ligand removal mainly controls the AuNP agglomeration, and that 2) $\mathrm{Na}_{2} \mathrm{CO}_{3}\left(\mathrm{CO}_{3}{ }^{2-}\right)$ on AuNPs are removed irreversibly by laser irradiation.
For the first point, by comparing the absorption spectra of AuNPs in $\mathrm{Na}_{2} \mathrm{CO}_{3}$ solutions before laser irradiation with those of AuNPs in citrate and $\mathrm{NaCl}$ solutions (Fig. 8), the shape of the plasmon band of AuNPs in $\mathrm{Na}_{2} \mathrm{CO}_{3}$ solutions is more similar to that of AuNPs in citrate solutions than that of AuNPs in $\mathrm{NaCl}$ solutions. This result supports that the agglomeration conditions of AuNPs in $\mathrm{Na}_{2} \mathrm{CO}_{3}$ solutions before laser irradiation are more similar to that of AuNPs in citrate solutions than that of AuNPs in $\mathrm{NaCl}$ solutions, and that laser-induced removal of $\mathrm{Na}_{2} \mathrm{CO}_{3}\left(\mathrm{CO}_{3}{ }^{2-}\right)$ mainly controls the AuNP agglomeration in $\mathrm{Na}_{2} \mathrm{CO}_{3}$ solutions. Such difference in the agglomeration conditions of AuNPs will be attributed to the difference in the stabilization ability of these reagents. A possible factor that determines the stabilization ability of the reagents might be the valence number of the ions. A citrate ion, $\mathrm{a} \mathrm{CO}_{3}{ }^{2-}$ ion, and a $\mathrm{Cl}^{-}$ion respectively has $3 \mathrm{e}^{-}, 2 \mathrm{e}^{-}$, and $\mathrm{e}^{-}$negative charges. The stabilization ability of the reagents will increase with the increasing negative charge. However, further investigation of the stabilizing ability of ions should be carried out on the basis of various properties of ions [14].

For the second point, considering that the irreversible removal of citrate ions and the reversible removal of $\mathrm{Cl}^{-}$ions have been respectively explained in terms of decomposition of citrate ions and non-decomposition of $\mathrm{Cl}^{-}$ions by laser irradiation, the irreversible removal of $\mathrm{CO}_{3}{ }^{2-}$ ions in

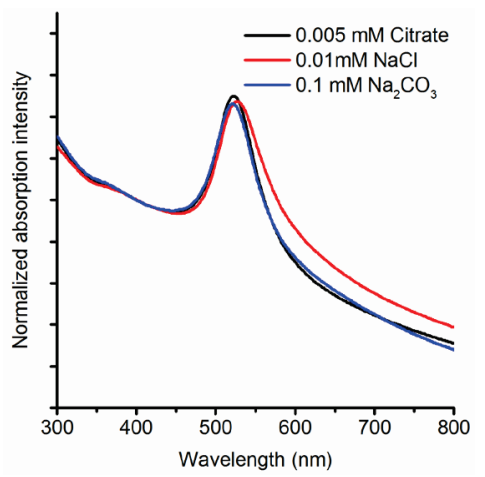

Fig. 8 Comparison of absorption spectra of the source AuNPs in $0.1 \mathrm{mM} \mathrm{Na} \mathrm{CO}_{3}, 0.01 \mathrm{mM}$ sodium citrate, and $0.05 \mathrm{mM} \mathrm{NaCl}$. 
(a)

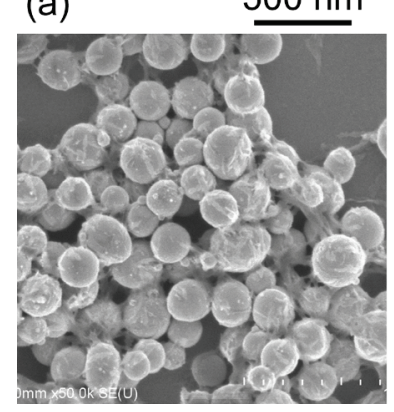

(b)

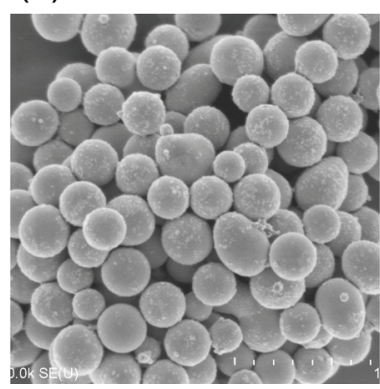

(c)

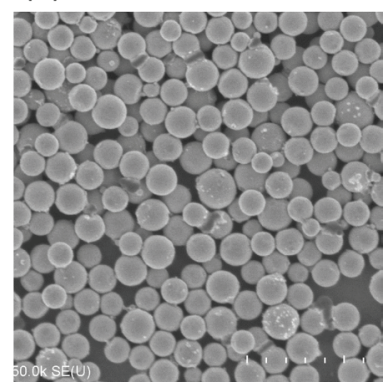

Fig. 9 SEM images of AuSMPs prepared by LML using AuNPs stabilized by (a) $0.01 \mathrm{mM} \mathrm{Na}_{2} \mathrm{CO}_{3}$, (b) $0.005 \mathrm{mM}$ citrate, and (c) $0.01 \mathrm{mM} \mathrm{NaCl}$. The average diameter of the AuSMPs are (a) $197 \mathrm{~nm}$, (b) $234 \mathrm{~nm}$, and (c) $146 \mathrm{~nm}$. The laser fluence used in LML was $60 \mathrm{~mJ} / \mathrm{cm}^{2}$. The LAL conditions used to prepare the AuNPs were, fluence: $36 \mathrm{~J} / \mathrm{cm}^{2}$ and duration: $20 \mathrm{~min}$.

LML using $\mathrm{Na}_{2} \mathrm{CO}_{3}$ suggests that $\mathrm{CO}_{3}{ }^{2-}$ ions will be decomposed by laser irradiation, although no evidence has been obtained at present.

The size of AuSMPs produced from AuNPs stabilized by citrate, $\mathrm{NaCl}$, and $\mathrm{Na}_{2} \mathrm{CO}_{3}$ by LML at the same laser fluence $\left(60 \mathrm{~mJ} / \mathrm{cm}^{2}\right)$ was compared in Fig. 9. Theoretically, it is predicted that the size of AuSMPs obtained by LML is determined by fluence of laser irradiation [16]. However, Fig. 9 shows that the particle size depends on the species of stabilizing reagents. As reported previously [11], the smaller size of AuSMPs prepared using AuNPs stabilized by $\mathrm{NaCl}$ can be attributed to the inhibition of the agglomeration of AuNPs, i.e., the growth of AuSMPs, by the readsorption of $\mathrm{Cl}^{-}$ions on AuNPs. Assuming such effect, the fact that the size of AuSMPs prepared using $\mathrm{Na}_{2} \mathrm{CO}_{3}$ is larger than that of AuSMPs prepared using $\mathrm{NaCl}$ suggests that the inhibition of the agglomeration of AuNPs in $\mathrm{Na}_{2} \mathrm{CO}_{3}$ solutions occurs less efficiently than that in $\mathrm{NaCl}$ solutions, consistent with the assumption that $\mathrm{Na}_{2} \mathrm{CO}_{3}$ $\left(\mathrm{CO}_{3}{ }^{2-}\right)$ are irreversibly removed from AuNPs by laser irradiation. These findings indicate that stabilizing reagent is also an important factor to determine the particle size.

\section{Conclusion}

AuSMPs were successfully obtained by LML using AuNPs stabilized by $\mathrm{Na}_{2} \mathrm{CO}_{3}$. It was also found that the amount of remaining AuNPs can be reduced by adjusting the concentrations of $\mathrm{Na}_{2} \mathrm{CO}_{3}$ and AuNPs. The agglomeration-fusion process during laser irradiation was compared with those for LML using AuNPs stabilized by citrate and $\mathrm{NaCl}$. It was suggested that the formation process of AuSMPs in LML using AuNPs stabilized by $\mathrm{Na}_{2} \mathrm{CO}_{3}$ was similar to that in LML using AuNPs stabilized by citrate. It was also found that the AuSMPs size depends on stabilizing reagents. These findings indicate that stabilizing reagent is important factor to control the formation efficiency and size of AuSMPs. In addition, the results showed that fast agglomeration of the source AuNPs reduces the remaining of the AuNPs after LML, which will be important information for efficient preparation of AuSMPs and for precise control of the AuSMP size. We are continuing further investigation to clarify this phenomenon.

\section{Acknowledgments}

The authors thank Prof. Shuichi Hashimoto (Tokushima University) and Dr. Alexander Pyatenko (AIST) for useful discussions related to laser-induced heating of metal NPs in liquids. This research was financially supported by a Grantin-Aid for Scientific Research (C) (50284568) and by Management Expenses Grants for National University Corporations from the Ministry of Education, Culture, Sports, Science and Technology of Japan.

\section{References}

[1] Y. Ishikawa, Q. Feng, N. Koshizaki, Appl. Phys. A, 99 (2010) 797.

[2] H.Q. Wang, A. Pyatenko, K. Kawaguchi, X. Li, Z. Swiatkowska-Warkocka, N. Koshizaki, Angew. Chem. Int. Ed., 49 (2010) 6361.

[3] X.Y. Li, A. Pyatenko, Y. Shimizu, H.Q. Wang, K. Koga, N. Koshizaki, Langmuir, 27 (2011) 5076.

[4] H.Q. Wang, N. Koshizaki, L. Li, L.C. Jia, K. Kawaguchi, X.Y. Li, A. Pyatenko, Z. SwiatkowskaWarkocka, Y. Bando, D. Golberg, Adv. Mater., 23 (2011) 1865.

[5] H.Q. Wang, M. Miyauchi, Y. Ishikawa, A. Pyatenko, N. Koshizaki, Y. Li, L. Li, X.Y. Li, Y. Bando, D. Golberg, J. Am. Chem. Soc., 133 (2011) 19102.

[6] H.Q. Wang, K. Kawaguchi, A. Pyatenko, X.Y. Li, Z. Swiatkowska-Warkocka, Y. Katou, N. Koshizaki, Chem. Eur. J., 18 (2012) 163.

[7] Y. Ishikawa, Y. Katou, N. Koshizaki, Q. Feng, Chem. Lett., 42 (2013) 530.

[8] T. Tsuji, T. Yahata, M. Yasutomo, K. Igawa, M. Tsuji, Y. Ishikawa, N. Koshizaki, Phys. Chem. Chem. Phys., 15 (2013) 3099.

[9] C. Rehbock, J. Zwartscholten, S. Barcikowski, Chem. Lett., 43 (2014) 1502.

[10] H. Fujiwara, R. Niyuki, Y. Ishikawa, N. Koshizaki, T. Tsuji, K. Sasaki, Appl. Phys. Lett., 102 (2013) 061110.

[11] T. Tsuji, Y. Higashi, M. Tsuji, Y. Ishikawa, N. Koshizaki, Appl. Surf. Sci., 348 (2015) 10.

[12] J.P. Sylvestre, S. Poulin, A.V. Kabashin, E. Sacher, M. Meunier, J.H.T. Luong, J. Phys. Chem. B, 108 (2004) 16864.

[13] C. Rehbock, V. Merk, L. Gamrad, R. Streubel, S. Barcikowski, Phys. Chem. Chem. Phys., 15 (2013) 3057.

[14] V. Merk, C. Rehbock, F. Becker, U. Hagemann, H. Nienhaus, S. Barcikowski, Langmuir, 30 (2014) 4213.

[15] C.F. Bohren, D.R. Huffman: Absorption and scattering of light by small particles, Wiley, New York, 1998.

[16] A. Pyatenko, H.Q. Wang, N. Koshizaki, T. Tsuji, Laser Photon. Rev., 7 (2013) 596.

(Received: May 25, 2015, Accepted: December 11, 2015) 